\title{
Erratum to: Phosphocreatine protects against LPS-induced human umbilical vein endothelial cell apoptosis by regulating mitochondrial oxidative phosphorylation
}

\author{
Zhengwu Sun ${ }^{1,2} \cdot$ Xiaoyan $\mathrm{Lan}^{3} \cdot$ Anil Ahsan ${ }^{1}$ - Yalin $\mathrm{Xi}^{2} \cdot$ Shumin Liu ${ }^{1}$. \\ Zonghui Zhang $^{1} \cdot$ Peng Chu $^{1} \cdot$ Yushu Song ${ }^{1} \cdot$ Fengyuan Piao ${ }^{4} \cdot$ Jinyong Peng ${ }^{1}$. \\ Yuan Lin $^{1} \cdot$ Guozhu Han ${ }^{1}$ Zeyao Tang ${ }^{1}$
}

Published online: 29 January 2016

(C) Springer Science+Business Media New York 2016

\section{Erratum to: Apoptosis}

DOI 10.1007/s10495-015-1210-5

The original version of this article unfortunately contained a mistake. The arrow marks in Fig. 5 were incorrect. It is now corrected with this erratum. The correct version of Fig. 5 is given below. The authors apologise for this error and the inconvenience it has caused to the readers.

The online version of the original article can be found under doi:10.1007/s10495-015-1210-5.

Zeyao Tang

tangzeyao@aliyun.com

1 Department of Pharmacology, Dalian Medical University, West Section 9, South Road of Lvshun, Dalian 116044, China

2 Pharmacy Department, Dalian Municipal Central Hospital, Dalian, China

3 Neurology Department, Dalian Municipal Central Hospital, Dalian, China

4 Public Health Department, Dalian Medical University, Dalian, China 


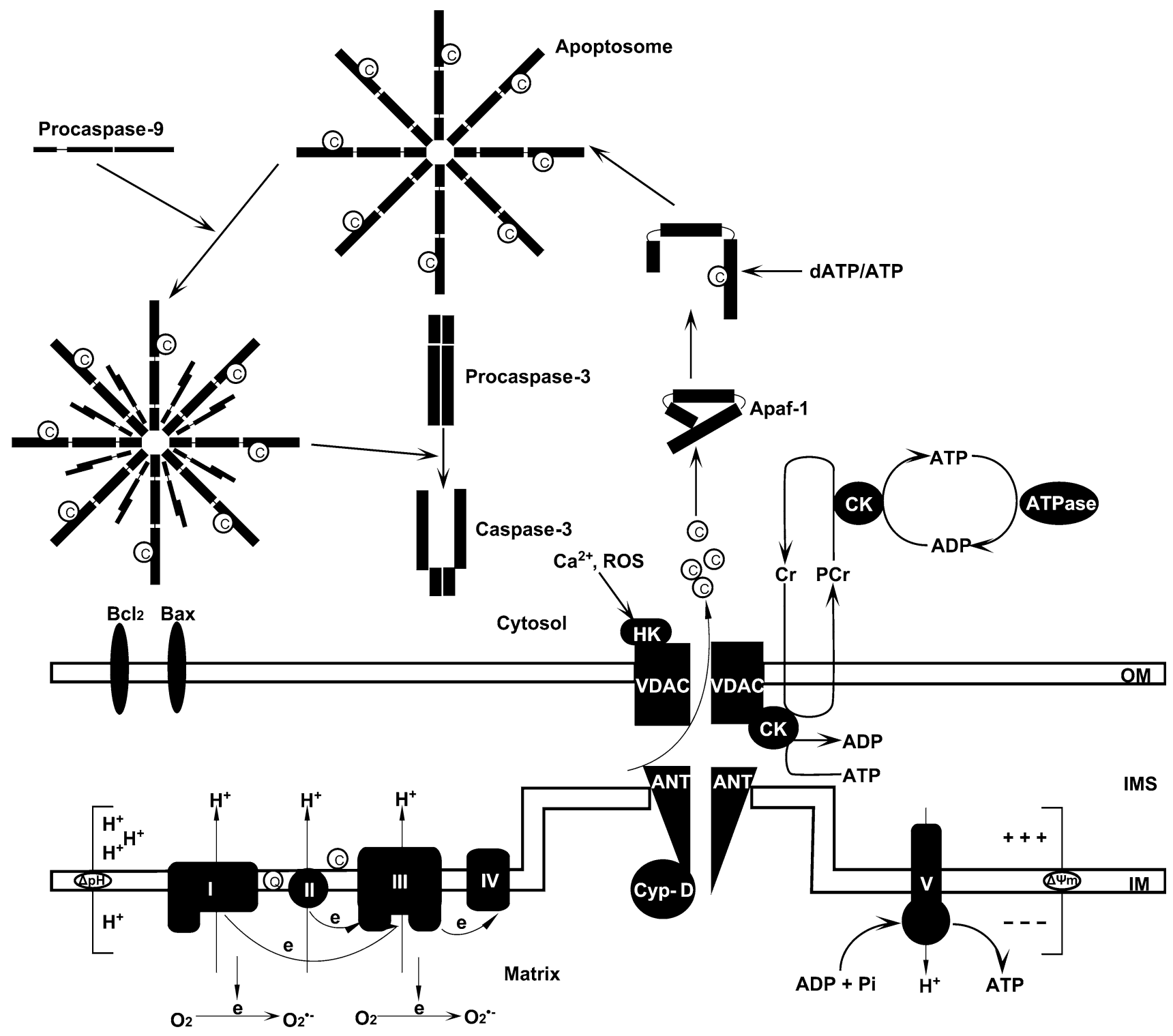

VDAC: Voltage-Dependent Anion Channe

-ANT: Adenine Nucleotide Translocase

Cyp-D: Cyclophilin-D

'HK: Hexokinase

'CK: Creatine Kinase

'I: Respiratory Chain Complexes I

'II: Respiratory Chain Complexes II

'III: Respiratory Chain Complexes III
-IV: Respiratory Chain Complexes IV

'V: FoF1- ATP Synthase

Q $Q$ : Coenzyme $Q$

'C: Cytochrome C

-OM: Outer Membrane

-IMS: Inner Membrane Space

-IM: Inner Membrane

Fig. 5 Schematic summary of the protection of ectogenic PCr against LPS-induced HUVECs mitochondrial apoptosis 